\title{
Video Article \\ Directed Cellular Self-Assembly to Fabricate Cell-Derived Tissue Rings for Biomechanical Analysis and Tissue Engineering
}

\author{
Tracy A. Gwyther ${ }^{1}$, Jason Z. Hu ${ }^{1}$, Kristen L. Billiar ${ }^{1}$, Marsha W. Rolle ${ }^{1}$ \\ ${ }^{1}$ Biomedical Engineering Department, Worcester Polytechnic Institute \\ Correspondence to: Marsha W. Rolle at mrolle@wpi.edu \\ URL: https://www.jove.com/video/3366 \\ DOI: doi:10.3791/3366
}

Keywords: Bioengineering, Issue 57, Cell-derived matrix, vascular tissue engineering, smooth muscle cells, cellular self-assembly, tissue biomechanics

Date Published: $11 / 25 / 2011$

Citation: Gwyther, T.A., Hu, J.Z., Billiar, K.L., Rolle, M.W. Directed Cellular Self-Assembly to Fabricate Cell-Derived Tissue Rings for Biomechanical Analysis and Tissue Engineering. J. Vis. Exp. (57), e3366, doi:10.3791/3366 (2011).

\section{Abstract}

Each year, hundreds of thousands of patients undergo coronary artery bypass surgery in the United States. ${ }^{1}$ Approximately one third of these patients do not have suitable autologous donor vessels due to disease progression or previous harvest. The aim of vascular tissue engineering is to develop a suitable alternative source for these bypass grafts. In addition, engineered vascular tissue may prove valuable as living vascular models to study cardiovascular diseases. Several promising approaches to engineering blood vessels have been explored, with many recent studies focusing on development and analysis of cell-based methods. ${ }^{2-5}$ Herein, we present a method to rapidly self-assemble cells into 3D tissue rings that can be used in vitro to model vascular tissues.

To do this, suspensions of smooth muscle cells are seeded into round-bottomed annular agarose wells. The non-adhesive properties of the agarose allow the cells to settle, aggregate and contract around a post at the center of the well to form a cohesive tissue ring. ${ }^{6,7}$ These rings can be cultured for several days prior to harvesting for mechanical, physiological, biochemical, or histological analysis. We have shown that these cell-derived tissue rings yield at $100-500 \mathrm{kPa}$ ultimate tensile strength ${ }^{8}$ which exceeds the value reported for other tissue engineered vascular constructs cultured for similar durations $(<30 \mathrm{kPa}){ }^{9,10}$ Our results demonstrate that robust cell-derived vascular tissue ring generation can be achieved within a short time period, and offers the opportunity for direct and quantitative assessment of the contributions of cells and cell-derived matrix (CDM) to vascular tissue structure and function.

\section{Video Link}

The video component of this article can be found at https://www.jove.com/video/3366/

\section{Protocol}

\section{Cell seeding mold fabrication}

Begin by milling a 1/2" thick piece of polycarbonate to create 15, round-bottomed, annular wells with a center post diameter of $2 \mathrm{~mm}$. The milled channels are $6 \mathrm{~mm}$ deep and $3.75 \mathrm{~mm}$ wide. Clean and dry the polycarbonate mold to remove any plastic debris from the milling process.

Mix polydimethylsiloxane (PDMS) at a 10:1 ratio (w/w) of base to curing agent, degas to remove all air bubbles, and pour onto the polycarbonate mold. Degas again to remove any remaining bubbles, and cure in the oven at $60^{\circ} \mathrm{C}$ for 4 hours.

Once cured, carefully remove the PDMS template by slowly peeling it away from the polycarbonate, wash with soap and water, and autoclave. Also autoclave a solution of two percent agarose (w/v) dissolved in Dulbecco's modified Eagle medium (DMEM).

Place the PDMS template on a level surface and fill with molten agarose by first pipetting agarose into each of the center post molds, and then pipetting into the space around it. Allow the agarose to solidify (approximately 15 minutes), then invert the mold to release the agarose from the PDMS. Cut excess agarose from around each of the wells, and place the agarose wells into 12-well plates.

\section{Cell culture and ring seeding}

Add cell culture media (DMEM with 10\% FBS and 1\% penicillin-streptomycin) around the outside of the agarose mold, without covering the top of the molds. Place the plates in the incubator and allow them to equilibrate for approximately 15 minutes while you prepare the cells.

At $90 \%$ confluence, trypsinize rat aortic smooth muscle cells (rSMCs) and resuspend at a concentration of $5 \times 10^{6}$ cells $/ \mathrm{ml}$. Pipette $100 \mu \mathrm{L}$ of cell suspension into each of the annular agarose wells, using a circular motion to apply cells to each well. 
Make sure incubator shelves are level, then place the plates in the incubator and allow them to sit undisturbed for 24 hours. Exchange medium every two days thereafter by aspirating the media from around the well, and re-filling each well until the agarose well is completely submerged.

\section{Tissue ring harvesting and thickness measurements}

At the conclusion of culture, remove the ring from the agarose mold by sliding it over the top of the center post.

Place the ring in a small Petri dish with phosphate buffered saline (PBS), center the sample, and acquire an image using a digital imaging system.

Use an image analysis program for edge detection and measure the thickness of the ring in 4 positions (top, bottom, left and right) around its circumference. Calculate the mean thickness value (t) for each individual ring, and use this value to calculate the cross-sectional area $\left(2 \pi(t / 2)^{2}\right)$.

\section{Mechanical testing of rings}

Set up the tensile testing machine (Instron EPS 1000) in the horizontal position. Attach a $1 \mathrm{~N} \pm 1 \mathrm{mN}$ load cell and custom thin wire grips (created by bending stainless steel wire).

Mount the ring sample on the two thin wire grips. Extend the grips until a $5 \mathrm{mN}$ tare load is applied to the sample. Enter the cross-sectional area (calculated from thickness measurements) and record the gauge length.

Pre-cycle the rings 8 times between the tare load and $50 \mathrm{kPa}$ stress at a rate of $10 \mathrm{~mm} / \mathrm{min}$. After the eighth pre-cycle, pull to failure at $10 \mathrm{~mm} /$ $\min$. Failure is noted as a decrease in force by $40 \%$ from one measurement to the next. For each ring sample, measure the ultimate tensile stress (UTS) and failure strain and calculate the maximum tangent modulus (MTM) from the acquired data.

\section{Tissue ring assembly to fabricate tissue tubes}

Custom tube holders are machined from round $5 \mathrm{~cm}$ polycarbonate disks with rectangular cutouts $(2 \mathrm{~cm} \times 3 \mathrm{~cm})$. Drill threaded holes through these disks to allow two holders to be screwed together. Autoclave the custom tube holders, and then transfer to the biosafety cabinet and place in an empty Petri dish

Use surgical scissors to cut the ends of $1.9 \mathrm{~mm}$ diameter silicone tubes at an angle to create beveled edges, and then autoclave the silicone tubes. While the tubes are being autoclaved, fill a Petri dish with media.

Remove the rings from the agarose wells and place them in the Petri dish with media. Place autoclaved silicone tubes in the same Petri dish to wet them before use.

Use forceps to place a beveled end of a silicone tube into the center of a ring and gently slide the ring onto the silicone tubing. Repeat with the desired number of rings. Slide the rings into contact with one another by gently pushing them successively in both directions along the tube. This method can be used for rings harvested after only one day in culture.

Once rings are mounted, align silicone tubes within the polycarbonate holders and screw the two parts of the holder together. Place the holder in a $100 \mathrm{~mm}$ Petri dish and add $55 \mathrm{~mL}$ media. Exchange media every 3 days for the duration of culture.

To remove the tissue tubes, release the silicone tubes from the polycarbonate holder and use forceps to slide the tissue tubes off the silicone tube and into a Petri dish filled with PBS.

\section{Representative Results:}

When the protocol is performed correctly, cells aggregate to form tissue rings with an inner diameter equal to the diameter of the corresponding mold post within 24 hours. The ring edges are usually smooth in appearance and (if cultured on a level surface), are uniform in thickness around the entire circumference. The tissue rings are easy to handle and can be removed from their wells for subsequent mechanical and histological analysis (see in Gwyther et al., 2011 ${ }^{8}$ ). Tissue ring morphology, matrix composition, and mechanical properties vary depending on the number and type of cells seeded.

Representative results from tissue rings made from varying cell types, seeding conditions, and culture length are shown in Table 1 . Two mm ID rings created from $5 \times 10^{5}$ rSMCs exhibited a higher UTS than rings created from our previously published 2 mm rings (made from $6.6 \times 10^{5}$ rSMCs). ${ }^{8}$ Similar to rat cells, human smooth muscle cells (hSMC) readily aggregated to form tissue rings, which contained a large amount of collagen after only 14 days in culture (data not shown). Human mesenchymal stem cells (hMSC) also aggregated and formed cohesive rings, but lacked mechanical strength and broke during the initial precycling phases of uniaxial tensile testing.

\begin{tabular}{|l|l|l|l|l|l|l|l|}
\hline & $\mathrm{n}$ & Cell number & Thickness $(\mathrm{mm})$ & $\begin{array}{l}\text { Length of culture } \\
(\mathrm{days})\end{array}$ & UTS $(\mathrm{kPa})$ & $\begin{array}{l}\mathrm{MTM}(\mathrm{kPa}) \\
(\mathrm{mm} / \mathrm{mm})\end{array}$ \\
\hline rSMC rings & 6 & 660,000 & $0.94 \pm 0.12$ & 14 & $97 \pm 30$ & $497 \pm 91$ & $0.50 \pm 0.08$ \\
\hline rSMC rings & 4 & 500,000 & $0.53 \pm 0.02$ & 7 & $113 \pm 8$ & $189 \pm 15$ & $0.88 \pm 0.05$ \\
\hline hSMC rings & 3 & 750,000 & $0.51 \pm 0.05$ & 14 & $160 \pm 30$ & $270 \pm 20$ & $0.92 \pm 0.08$ \\
\hline
\end{tabular}




\begin{tabular}{|l|l|l|l|l|l|l|l|}
\hline hMSC rings & 3 & 750,000 & $0.40 \pm 0.07$ & 14 & N/A & N/A \\
\hline
\end{tabular}

Table 1. Table showing the culture parameters and mechanical properties of $2 \mathrm{~mm}$ rings generated from different cell types, seeding concentrations, and culture durations.

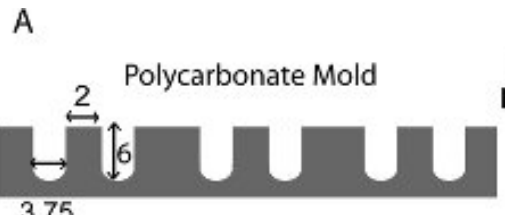

3.75

Separate individual molds
Cast PDMS

template on

polycarbonate mold

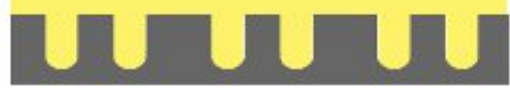

\section{Cast agarose \\ wells from}

PDMS template
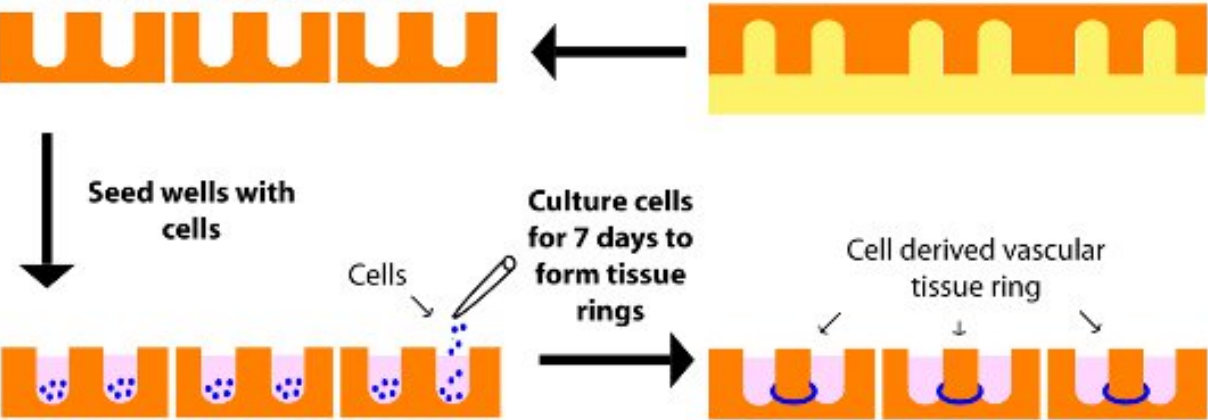

Culture cells

for 7 days to

form tissue

Cell derived vascular tissue ring

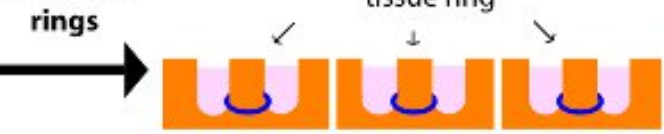

B

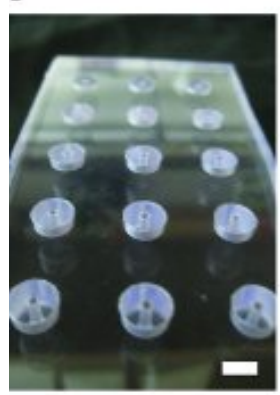

C

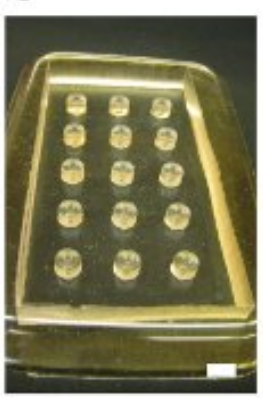

PDMS template

D

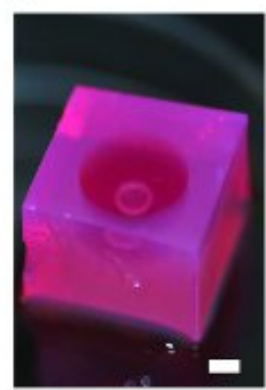

Tissue ring in agarose well
E

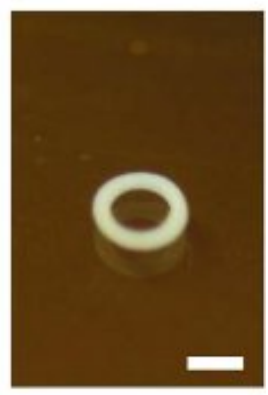

Tissue ring removed from well

Figure 1. (A) Schematic of the tissue ring generation process. (B) Custom polycarbonate mold with milled annular wells. Central post diameters are $2 \mathrm{~mm}$. (C) PDMS template after it was peeled away from the polycarbonate mold. (D) Aggregated tissue ring cultured in an agarose mold with a $2 \mathrm{~mm}$ post. (E) Two $\mathrm{mm}$ diameter tissue ring in PBS. Scale bars $=6 \mathrm{~mm}(B, C)$ and $2 \mathrm{~mm}(\mathrm{D}, \mathrm{E})$. 


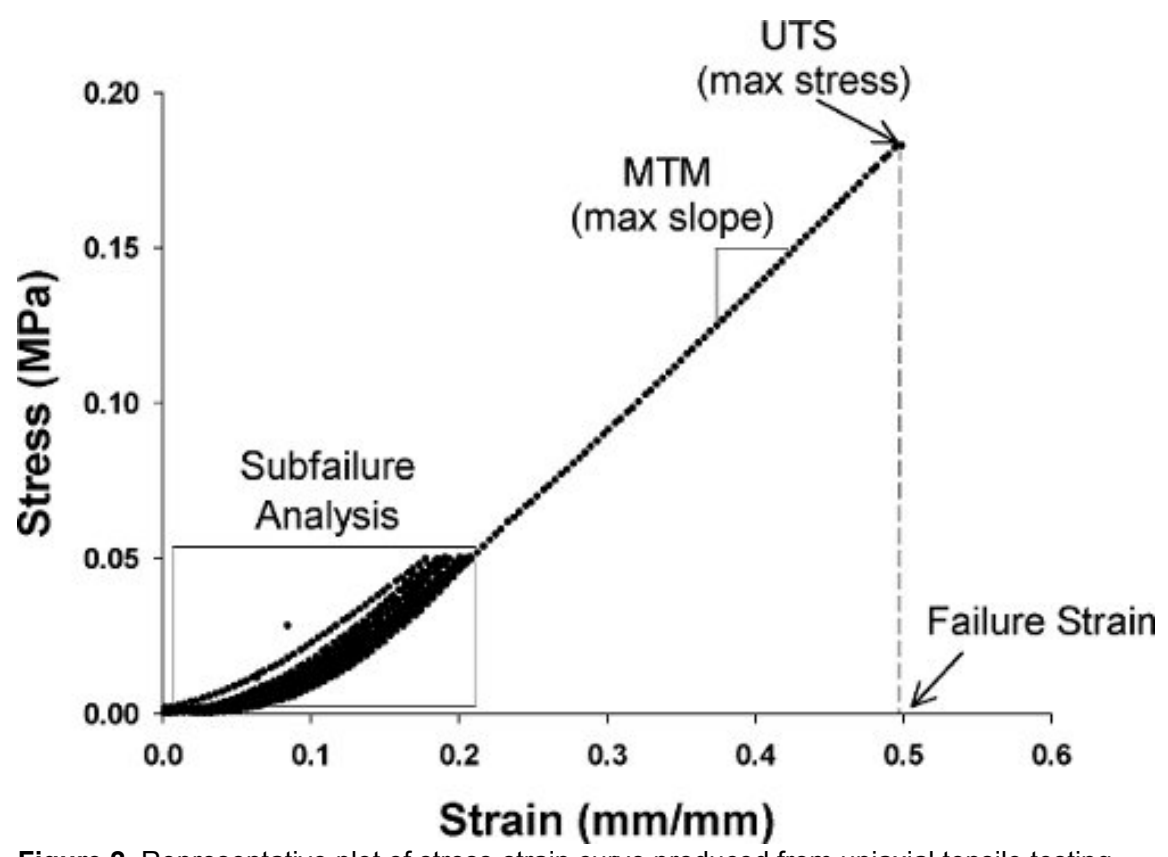

Figure 2. Representative plot of stress-strain curve produced from uniaxial tensile testing.

\section{Discussion}

Recently, there has been an increased interest in cell-based or "scaffold-less" tissue engineering methods to address some of the limitations of scaffold-based tissue engineering approaches. Given that cell-derived tissues are created from cells and the matrix they produce, they inherently contain much higher cell densities, contain no exogenous materials, and can be made entirely from human cells and proteins. Vascular grafts made from human cells can attain substantial mechanical strength in the absence of exogenous scaffolds (e.g., burst pressures $3400 \mathrm{mmHg}$ compared to $1600 \mathrm{mmHg}$ for human saphenous veins) $)^{12}$. Although cell-based vascular tissues exhibit improved cell density and mechanical strength, most current fabrication methods (such as "sheet-based engineering"3,4,12 or "bioprinting"5,13) require extensive culture periods (> 3 months) or specialized equipment for 3D tissue construction. The cell-derived tissue ring method described here enables rapid cellular selfassembly to form robust 3D tissue constructs within a short period of time and without the use of specialized equipment.

This protocol details the procedure we developed to create $2 \mathrm{~mm}$ inner diameter rat smooth muscle cell-derived tissue rings. In the present example, tissue rings were cultured for 7 days (then cultured for an additional 7 days for ring fusion and tube formation). However, $2 \mathrm{~mm}$ rat (and human) smooth muscle cell rings can be removed from the wells and are cohesive enough for handling (e.g., transfer onto silicone tubes) as early as one day after cell seeding. In addition, robust tissue rings with varying inner diameters $(2,4$, and $6 \mathrm{~mm})$ can be created with this method by simply changing the post diameter of the original polycarbonate mold. ${ }^{8}$ We also recently modified the polycarbonate mold design to enable five $2 \mathrm{~mm}$ seeding wells to be cast in a single multi-well agarose chamber, which uses less PDMS and agarose, and fits in one well of a 6-well plate (data not shown). Changes in the post diameter, the width of the seeding well, the radius of curvature of the rounded bottom, the number of seeding wells, or the depth of the seeding wells can all be modified simply by changing the specifications in the CAD file for CNC machining of the polycarbonate mold. Finally, a single polycarbonate mold can be used to fabricate an unlimited number of PDMS templates, and each PDMS template can be cleaned, autoclaved and reused dozens of times.

In addition to changing the size of the tissue rings, we have made rings from many different cell types, including: primary rat SMCs (Cell Applications, R354-05), primary human coronary artery SMCs (Lonza, CC-2583), primary human dermal fibroblasts ${ }^{11}$ (generous gift of Dr. George Pins, WPI Dept. of Biomedical Engineering), rat lung fibroblasts (RFL-6, ATCC CCL-192), and mesenchymal stem cells (Lonza, PT-2501). Each of these cell types aggregates and contracts around the center posts to form tissue rings; although the cellular organization, ECM composition, and mechanical properties of the constructs vary for each cell type. The seeding parameters for each cell type must be empirically determined based on the size of the cells and their ability to aggregate. Therefore, while this system of creating cell-derived tissue rings is extremely versatile, the protocol may need slight adjustments for optimal tissue formation with different cell types.

Tissue ring geometry facilitates easy loading and assessment of tissue material properties by uniaxial tensile testing, as described. There is also substantial precedent for using blood vessel ring segments to measure vascular contraction and physiological function. Preliminary studies indicate that cell-derived tissue rings can be mounted on a wire myograph device for measurement of pharmacological responsiveness and contractile force generation (data not shown). Altogether, the ability to rapidly fabricate self-assembled cell rings for histological, mechanical, physiological, and biochemical analysis suggests a powerful new tool that may be useful for modeling vascular tissue structure and function in health and disease.

\section{Disclosures}

No conflicts of interest declared. 


\section{Acknowledgements}

The authors gratefully acknowledge Neil Whitehouse (WPI, Higgins Machine Shop) for his assistance with CNC machining. Additionally, we would like to thank Adriana Hera (WPI Computing and Communications Center) for her help with MATLAB programming, as well as Kate Beverage and Joseph Cotnoir (WPI Academic Technology Center) for assistance with Camtasia. Sophie Burke and Jacleen Becker (WPI Academic Technology Center) provided supplemental video footage. This work was funded by the National Institutes of Health (R15 HL097332), the UMass Medical School-WPI Pilot Research Initiative, the American Heart Association (undergraduate research fellowship to J.Z.H), and Worcester Polytechnic Institute (Summer Undergraduate Research Fellowship to J.Z.H. and institutional start-up funds to M.W.R.).

\section{References}

1. Roger, V.L., et al. Heart Disease and Stroke Statistics--2011 Update: A Report From the American Heart Association. Circulation. 123, e18e209 (2011).

2. Kelm, J. M., et al. A novel concept for scaffold-free vessel tissue engineering: self-assembly of microtissue building blocks. J. Biotechnol. 148, 46-55 (2010).

3. Gauvin, R., et al. A novel single-step self-assembly approach for the fabrication of tissue-engineered vascular constructs. Tissue. Eng. Part. A. 16, 1737-1747 (2010).

4. L'Heureux, N., McAllister, T.N., \& de la Fuente, L.M. Tissue-engineered blood vessel for adult arterial revascularization. N. Engl. J. Med. 357, 1451-1453 (2007).

5. Norotte, C., Marga, F.S., Niklason, L.E., \& Forgacs, G. Scaffold-free vascular tissue engineering using bioprinting. Biomaterials. $\mathbf{3 0}$, 5910-5917 (2009).

6. Dean, D.M., Napolitano, A.P., Youssef, J., \& Morgan, J.R. Rods, tori, and honeycombs: the directed self-assembly of microtissues with prescribed microscale geometries. FASEB. J. 21, 4005-4012 (2007).

7. Livoti, C.M. \& Morgan, J.R. Self-assembly and tissue fusion of toroid-shaped minimal building units. Tissue. Eng. Part. A. 16, 2051-2061 (2010).

8. Gwyther, T., et al. Engineered vascular tissue fabricated from aggregated smooth muscle cells. Cell. Tissues. Organs. 194, 13-24 (2011).

9. Seliktar, D., Black, R.A., Vito, R.P., \& Nerem, R.M. Dynamic mechanical conditioning of collagen-gel blood vessel constructs induces remodeling in vitro. Ann. Biomed. Eng. 28, 351-362 (2000).

10. Rowe, S.L. \& Stegemann, J.P. Interpenetrating collagen-fibrin composite matrices with varying protein contents and ratios. Biomacromolecules. 7, 2942-2948 (2006).

11. Pins, G.D., Collins-Pavao, M.E., Van De Water, L., Yarmush, M.L., \& Morgan, J.R. Plasmin triggers rapid contraction and degradation of fibroblast-populated collagen lattices. J. Invest. Dermatol. 114, 647-653 (2000).

12. Konig, G., et al. Mechanical properties of completely autologous human tissue engineered blood vessels compared to human saphenous vein and mammary artery. Biomaterials. 30, 1542-1550 (2009).

13. Mironov, V., et al. Organ printing: tissue spheroids as building blocks. Biomaterials. 30, 2164-2174 (2009). 